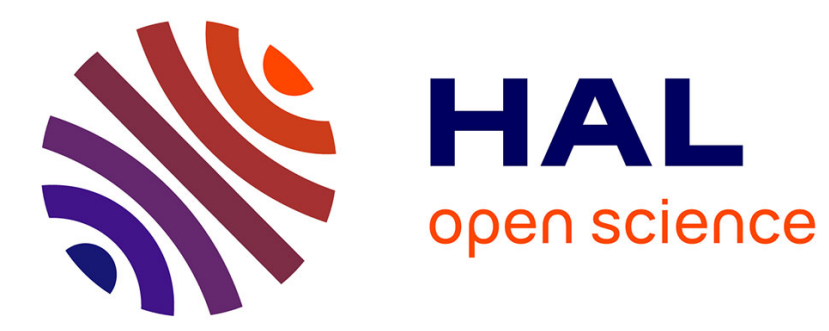

\title{
Graph-based inter-subject classification of local fMRI patterns
}

Sylvain Takerkart, Guillaume Auzias, Bertrand Thirion, Daniele Schön, Liva

Ralaivola

\section{- To cite this version:}

Sylvain Takerkart, Guillaume Auzias, Bertrand Thirion, Daniele Schön, Liva Ralaivola. Graph-based inter-subject classification of local fMRI patterns. Third International Workshop Machine Learning in Medical Imaging - MLMI 2012 (Held in Conjunction with MICCAI 2012), Oct 2012, Nice, France. pp 184-192, 10.1007/978-3-642-35428-1_23 . hal-00818815

\section{HAL Id: hal-00818815 https://hal.science/hal-00818815}

Submitted on 29 Apr 2013

HAL is a multi-disciplinary open access archive for the deposit and dissemination of scientific research documents, whether they are published or not. The documents may come from teaching and research institutions in France or abroad, or from public or private research centers.
L'archive ouverte pluridisciplinaire HAL, est destinée au dépôt et à la diffusion de documents scientifiques de niveau recherche, publiés ou non, émanant des établissements d'enseignement et de recherche français ou étrangers, des laboratoires publics ou privés. 


\title{
Graph-based inter-subject classification of local fMRI patterns
}

\author{
Sylvain Takerkart ${ }^{1,2}$, Guillaume Auzias ${ }^{3,1}$, Bertrand Thirion ${ }^{4}$, Daniele Schön ${ }^{5}$, \\ and Liva Ralaivola ${ }^{2}$ \\ ${ }^{1}$ CNRS, INT (UMR 7289), Marseille, France \\ 2 Aix-Marseille University, LIF (UMR 7189), Marseille, France \\ 3 CNRS, LSIS (UMR 7296), Marseille, France \\ 4 INRIA-Saclay-Ile-de-France, Parietal team, Palaiseau, France \\ ${ }^{5}$ CNRS, INS (UMR 1106), Marseille, France
}

\begin{abstract}
Classification of medical images in multi-subjects settings is a difficult challenge due to the variability that exists between individuals. Here we introduce a new graph-based framework designed to deal with inter-subject functional variability present in fMRI data. A graphical model is constructed to encode the functional, geometric and structural properties of local activation patterns. We then design a specific graph kernel, allowing to conduct SVM classification in graph space. Experiments conducted in an inter-subject classification task of patterns recorded in the auditory cortex show that it is the only approach to perform above chance level, among a wide range of tested methods.
\end{abstract}

Keywords: fMRI, classification, graphs, kernels, inter-subject, variability

\section{Introduction}

Since brain functions are considered to arise from activities distributed across networks, multivariate pattern recognition methods are adapted to study information processing in the brain. Multi-voxel pattern analysis (MVPA) of functional MRI allows to study the organization of distributed representations in the brain by using a classification framework where one attempts to predict the category of the input stimuli from the data [8]. MVPA has been largely applied within individual subjects [13].

However, because of the challenge posed by the large inter-individual variability, very few studies describe inter-subject decoding, i.e successfull prediction on data from a subject that was not part of the training set. Among these, most rely on global (i.e full-brain) analysis, using large-scale features ([12], [15]). The few studying fine-scale local patterns achieve very low inter-subject generalization performances [3], or use implicit abstract models [9]. None attempt to characterize the inter-subject functional variability (i.e the fact that the correlation between cortical folding and the underlying functional organization vary between subjects [4]) and use such characterization in the classification process. 
In this paper, we introduce a new framework specifically aimed at tackling the challenges offered by inter-subject functional variability by modelling its spatial properties. Our approach integrates the fact that the geometric properties of local functional features, as well as their levels of activation, can vary across subjects, under the assumption that the underlying spatial structure of the local activation pattern is consistent. We therefore design a graphical model to represent such patterns with their properties of interest: the nodes of the graphs represent small activation patches; their attributes carry the relevant features, such as their position and activation level; the edges of the graph (given by spatial adjacency) encode the spatial structure of the pattern. The classification is then performed directly in graph-space with Support Vector Machines (SVM), using a graph kernel specifically designed to take into account all properties of our graphical model. Since the topographic organization of primary sensory areas ensures the structural consistency of activation patterns across subjects, we validate our framework on fMRI data recorded in the primary auditory cortex during a tonotopy experiment [10].

This paper is organized as follows: we detail the construction of our graphical model in section 2 and the kernel design in section 3; we then describe our experiments in section 4 and present our results in section 5 .

\section{Graphical Model of Activation Patterns}

We here describe the design of a graphical model that summarizes the information relevant to characterize a pattern a pattern of activation measured with fMRI within a contiguous region of interest (ROI).

Graph nodes. Assuming that the ROI admits an underlying subdivision into a set of smaller and functionally relevant sub-regions, the first step to construct our graphical model estimates a parcellation of the ROI, i.e. a partition into a set of sub-regions or parcels [5]. Specifically, for a contiguous ROI $\mathcal{R}$, we compute the parcellation $\mathcal{V}=\left\{V_{i}\right\}_{i=1}^{q}$ of $\mathcal{R}$, so that the $q$ parcels verify: $\cup_{i=1}^{q} V_{i}=\mathcal{R}$ and $V_{i} \cap V_{j}=\emptyset$ whenever $i \neq j$. We use $\mathcal{V}$ as the set of vertices of our graphical model, each parcel corresponding to a node of the graph.

Nodes attributes: functional features. Let $f$ be the real-valued function describing the BOLD activation. In a parcel $V_{i}$, the activations values $\{f(v)\}_{v \in V_{i}}$ are summarized to form an $n$-dimensional feature vector $F\left(V_{i}\right)$; we note $\mathcal{F}$ the $n \times q$ matrix $\mathcal{F}=\left[F\left(V_{1}\right) \cdots F\left(V_{q}\right)\right]$ of functional features, where each column $i$ of $F$ is $F\left(V_{i}\right)$. A simple example of such $F$, which we make use of later on, is to compute the mean activation value within a parcel.

Nodes attributes: geometric features. Let $x$ be a coordinate system defined in $\mathcal{R}$. We summarize the geometric information of parcel $V_{i}$ through a $m$ dimensional feature vector $X\left(V_{i}\right)$, computed from the locations $\{x(v)\}_{v \in V_{i}}$. We note $\mathcal{X}$ the $m \times q$ matrix $\mathcal{X}=\left[X\left(V_{1}\right) \cdots X\left(V_{q}\right)\right]$ of geometric features. $\mathcal{X}$ may contain information on the shape or location of the parcels.

Graph edges: structural information. The set of edges is represented by a binary adjacency matrix $A=\left(a_{i j}\right) \in \mathbb{R}^{q \times q}$, where $a_{i j}=1$ if parcels $V_{i}$ and $V_{j}$ 
are spatially adjacent (i.e if $\exists v_{i} \in V_{i}, \exists v_{j} \in V_{j}$ so that $v_{i}$ and $v_{j}$ are neighbors, for a given neighborhood definition), and $a_{i j}=0$ otherwise. This adjacency matrix encodes the spatial structure of the activation pattern.

Full graphical model. Using these definitions, we have defined an attributed region adjacency graph [14] (hereafter noted $a-R A G) G=(\mathcal{V}, A, \mathcal{F}, \mathcal{X})$, which represents the fMRI activation pattern within the ROI $\mathcal{R}$ by encoding functional, geometric and structural information.

\section{Kernel design}

The fully generic family of convolution kernels [7] is defined as:

$$
K(G, H)=\sum_{g \subseteq G, h \subseteq H} \prod_{t} k_{t}(g, h),
$$

where $t \in \mathbb{N}^{*}$ is the, usually small, number of base kernels $k_{t}$, which act on subgraphs $g$ and $h$. To design a kernel for our a-RAGs, we need to choose the type of subgraphs, the value of $t$ and to instantiate each base kernel $k_{t}$.

Subgraphs used to design such kernels include paths and random walks [6], tree motives [11] etc. For simplicity reasons, we will here use paths of length two; note that the definitions below are directly extendable to other choices. Given the characteristics of the $a-R A G s$, we need to define $t=3$ elementary kernels $k_{f}$, $k_{g}$ and $k_{s}$, respectively acting on functional, geometric and structural features.

Let $G=\left(\mathcal{V}_{G}, A_{G}, \mathcal{F}_{G}, \mathcal{X}_{G}\right)$ and $H=\left(\mathcal{V}_{H}, A_{H}, \mathcal{F}_{H}, \mathcal{X}_{H}\right)$ be two a-RAGs: we note $g_{i j}=\{i, j\}$ and $h_{k l}=\{k, l\}$ two pairs of nodes in $G$ and $H$, respectively; let $q_{G}$ and $q_{H}$ be the number of nodes in $G$ and $H$, respectively - note that $q_{G}$ and $q_{H}$ may be different.

Functional kernel. Kernel $k_{f}$ aims at measuring the similarity of the activation in parcels of $g_{i j}$ and $h_{k l}$. We therefore propose the following product (and thus positive definite) kernel

$$
k_{f}\left(g_{i j}, h_{k l}\right)=e^{-\left\|F_{i}^{G}-F_{k}^{H}\right\|^{2} / 2 \sigma_{f}^{2}} \cdot e^{-\left\|F_{j}^{G}-F_{l}^{H}\right\|^{2} / 2 \sigma_{f}^{2}},
$$

where $\sigma_{f} \in \mathbf{R}_{+}^{*}$ and $F_{p}^{G}$ (resp. $F_{p}^{H}$ ) is the $p$ th column of $\mathcal{F}_{G}$ (resp. $\mathcal{F}_{H}$ ). Using such a product of Gaussian kernels allows dealing with the inter-subject functional variability.

Geometric kernel. The second base kernel $k_{g}$ acts on the geometric features. To allow for inter-subject variability, we follow the same principle as for the functional kernel, which gives:

$$
k_{g}\left(g_{i j}, h_{k l}\right)=e^{-\left\|X_{i}^{G}-X_{k}^{H}\right\|^{2} / 2 \sigma_{g}^{2}} \cdot e^{-\left\|X_{j}^{G}-X_{l}^{H}\right\|^{2} / 2 \sigma_{g}^{2}},
$$

where $\sigma_{g} \in \mathbf{R}_{+}^{*}$, and $X_{p}^{G}\left(\right.$ resp. $\left.X_{p}^{H}\right)$ is the $p$ th column of $\mathcal{X}_{G}$ (resp. $\mathcal{X}_{H}$ ).

Structural kernel. The base kernel $k_{s}$ aims at valuing the structural similarity of $G$ and $H$. Since our main hypothesis is that the structure is consistent across patterns recorded in different subjects, we adopt a decision function (by 
opposition to the smooth functional and geometric kernels), by using the linear kernel on binary entries $a_{i j}^{G}$ and $a_{k l}^{H}$ of the adjacency matrices $A_{G}$ and $A_{H}$, which encodes the fact that pairs $g_{i j}$ and $h_{k l}$ are both edges:

$$
k_{s}\left(g_{i j}, h_{k l}\right)=a_{i j}^{G} \cdot a_{k l}^{H}
$$

Resulting kernel. With the definitions of $k_{f}, k_{g}$ and $k_{s}$, we may define the resulting kernel (with parameters $\sigma_{g}$ and $\sigma_{f}$ ):

$$
K(G, H)=\sum_{i, j=1}^{q_{G}} \sum_{k, l=1}^{q_{H}} k_{g}\left(g_{i j}, h_{k l}\right) \cdot k_{f}\left(g_{i j}, h_{k l}\right) \cdot k_{s}\left(g_{i j}, h_{k l}\right),
$$
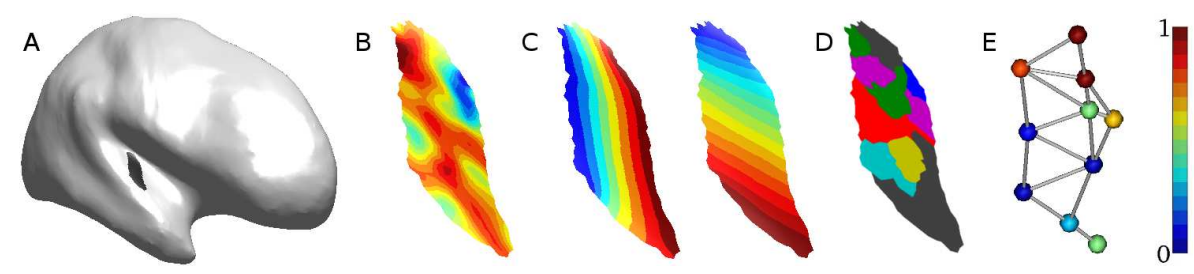

Fig. 1. Graph construction process. A. Inflated cortical surface of the right hemisphere for one subject; Heschl's gyrus is highlighted. B. A normalized activation pattern C. The two coordinates fields computed within the ROI. D. Example of parcellation with 10 parcels (arbitrary colors for illustration only). E. Example of an attributed graph: the nodes are located at the barycenter of the parcels and the color encodes the average level of activation in the parcel.

\section{Real experiment and data analysis}

fMRI tonotopy experiment. In order to test this framework, we used a dataset that was acquired to study the tonotopic property of the human auditory cortex. This property states that neighboring neurons in the cortex respond to auditory stimuli of neighboring frequencies. This results in a spatially organized mapping of the auditory cortex. Typically higher frequencies are represented in the medial part of the primary auditory cortex (A1) while lower frequencies are represented more laterally, although recent high resolution studies suggest a more complex patterns with mirror-symmetric frequency gradients [10].

For each of the nine subjects, a T1 image was acquired (1mm isotropic voxels). Each stimulus consisted of a 8 s sequence of 60 isochronous tones covering a narrow bandwidth around a central frequency $f$. There were five types of sequences (i.e. conditions), each one centered around a different frequency $f$ $(f \in\{300 \mathrm{~Hz}, 500 \mathrm{~Hz}, 1100 \mathrm{~Hz}, 2200 \mathrm{~Hz}, 4000 \mathrm{~Hz}\})$, with no overlap between the bandwidths covered by any two types of stimuli. Five functional sessions were 
acquired, each containing six sequences per condition presented in a pseudorandom order. Echo-planar images (EPI) were acquired with slices parallel to the sylvian fissure (repetition time $=2.4 \mathrm{~s}$, voxel size $=2 \times 2 \times 3 \mathrm{~mm}$, matrix size $128 \times 128$ ). Fieldmaps were recorded to allow EPI geometric distortion correction.

Data processing and classification. The preprocessing of the functional data, carried on in SPM8 (www.fil.ion.ucl.ac.uk/spm), consisted in realignment, slice timing correction and "fieldmap" unwarping. Then, a generalized linear model was performed (in nipy: nipy.sourceforge.net) with one regressor of interest per stimulus. The weight (beta) maps of these regressors served as estimates of the response size for each stimulus. For the spatial proximity to have an anatomical meaning, one has to work on the $2 \mathrm{D}$ cortical surface. We therefore used freesurfer (surfer.nmr.mgh.harvard.edu) to extract the cortical surface from the T1 image and automatically define the primary auditory cortex (Fig. 1A) as part of Heschl's gyrus (thus defining our cortical ROI $\mathcal{R}$ in each subject). The beta maps are then projected onto the cortex, and slightly smoothed (equivalent fwhm of $3 \mathrm{~mm}$ ) in cortical space, which defines the function $\{f(v)\}_{v \in \mathcal{R}}$ (Fig. 1B). A 2D local coordinates system is defined through a conformal mapping of $\mathcal{R}$ onto a rectangle [1], defining $\{x(v)\}_{v \in \mathcal{R}}$ (Fig. 1C). One then need a parcellation technique that produces homogeneous parcels. We use Ward's hierarchical clustering algorithm on anatomo-functional features $\{f(v), x(v)\}$, with an added spatial constraint: the merging criterion of two adjacent parcels consists in minimzing the variance across all parcels [12] (Fig. 1D), and the neighboring criterion is given by the neighboring of the vertices of the cortical mesh. Finally, we define one functional feature $F$, the mean activation in a parcel (normalized between 0 and 1) and a 2D geometric feature vector $X$ as the coordinates of the barycenter of each parcel.

Using the kernel trick, one can then directly perform Support Vector Classification in graph space (G-SVC) to guess the class of the input stimulus (i.e the tone frequency) from a given activation pattern. In practice, the Gram matrix was computed with custom-designed code, and given as input to the SVC function of the scikit-learn python module (scikit-learn.org), which uses Lib$S V M$ (www.csie.ntu.edu.tw/ cjlin/libsvm/) to implement SVM. A leaveone-subject-out cross-validation scheme was used to measure the classification accuracy. For each fold of this cross-validation, we estimated the kernel parameters $\sigma_{f}$ and $\sigma_{g}$ as the median euclidean distance between the functional and geometric (respectively) feature vectors of the parcels between all nodes of all example-graphs in the training set, which corresponds to a standard heuristic [2]. Two types of analyses were conducted. First, we performed simulations with a fixed number of nodes $q$ for all graphs and all subjects; several simulations were conducted, with $q \in\{5,6,7,8,9,10,15,20,25,30\}$. Second, we imposed different number of nodes for each subject, randomly taken in three intervals $I_{q}=[5,13]$, $[14,22]$ and $[5,21]$; for each of these intervals, we ran 18 simulations with different random sets of node numbers (each time with a different $q$ for each subject).

In order to compare the performances of our framework to vector-based methods, one need to define a vertex to vertex correspondance across subjects (which 
is not needed with our framework). We therefore aligned the anatomy of all subjects to a standard spherical cortical space using freesurfer and projected the beta maps to this standard space. $\mathcal{R}$ is now common to all subjects and we can use $\{f(v)\}_{v \in \mathcal{R}}$ as a feature set. Several classification algorithms were then used, each time with several values of their respective hyper-parameters: 1) linear SVC; 2) non linear SVC, with gaussian (with $\sigma \in\left\{10^{-n}\right\}_{n \in\{-2,-1,0,1\}}$ ) and polynomial (of order $n \in\{2,3,4\}$ ) kernels; 3) $k$-nearest neigbors (with $k \in\{3,5,7,11,15\}) ; 4)$ logistic regression with $l_{1}$ and $l_{2}$ regularization (with weight $\left.\lambda \in\left\{10^{n}\right\}_{n \in\{2,3,4\}}\right)$.

\section{Results and discussion}

Table 1 contains the performances of our G-SVC framework vs. the different benchmark vector-based methods. The scores are the mean classification accuracies across all folds of the cross-validation; for the benchmark methods, the reported score is the highest one across simulations ran with all values of their hyper-parameters; for G-SVC, it is the mean performances across all values of $q$. All vector-based methods performed similarly, but none of them performed significantely above chance level (equal to 0.2 since there were five types of auditory stimuli). Our G-SVC framework was the only method to perform significantely above chance level (one sample t-test, $p<0.05$, indicated by a $\star$ ), in both the left and right Heschl's gyri (HG). It also performed significantely better than the best benchmark method (linear SVC for the right HG, logistic regression for the left HG; paired t-test with matched left-out subject, $p<0.05$; indicated by $\mathrm{a} \diamond$ ). Table 2 describes the accuracy of our G-SVC depending on the number of graph nodes $q$. Our framework performed above chance level in all cases (one sample t-test, $p<0.05 ; \star)$, and significantely better than the best benchmark method (paired t-test, $p<0.05 ; \diamond$ ) in all but two cases (left HG, $q=10,15$ ).

\begin{tabular}{|c|c|c|c|c|c|}
\hline & G-SVC & lin. SVC & non lin. SVC & k-NN & log. reg. \\
\hline \hline right HG & $\mathbf{0 . 4 5}^{\star \diamond}$ & 0.25 & 0.23 & 0.27 & 0.27 \\
\hline left HG & $\mathbf{0 . 3 6}^{\star \diamond}$ & 0.26 & 0.24 & 0.25 & 0.24 \\
\hline
\end{tabular}

Table 1. G-SVC vs. benchmark methods

\begin{tabular}{|c|c|c|c|c|c|c|c|c|c|c|}
\hline G-SVC $q$ nodes & 5 & 6 & 7 & 8 & 9 & 10 & 15 & 20 & 25 & 30 \\
\hline \hline right HG & $\mathbf{0 . 4 8}^{\star \diamond}$ & $0.45^{\star \diamond}$ & $0.45^{\star \diamond}$ & $0.43^{\star \diamond}$ & $0.47^{\star \diamond}$ & $0.43^{\star \diamond}$ & $0.44^{\star \diamond}$ & $\mathbf{0 . 4 8}^{\star \diamond}$ & $0.47^{\star \diamond}$ & $0.44^{\star \diamond}$ \\
\hline left HG & $0.33^{\star \diamond}$ & $0.37^{\star \diamond}$ & $0.35^{\star \diamond}$ & $0.38^{\star \diamond}$ & $0.35^{\star \diamond}$ & $0.35^{\star}$ & $0.34^{\star}$ & $0.37^{\star \diamond}$ & $\mathbf{0 . 3 9}^{\star \diamond}$ & $0.38^{\star \diamond}$ \\
\hline
\end{tabular}

Table 2. Influence of the number of graph nodes $q$ in G-SVC

Results of the simulations using a randomized number of nodes $q$ for each subject are given in Table 3. In all 108 simulations, our G-SVC framework performed above chance level (one sample t-test, $p<0.05, \star$ ). This shows that the proposed kernel is efficient for graphs having different number of nodes. Moreover, in $80 \%$ of the simulations (86/108), it outperformed the best benchmark method (paired t-test, $p<0.05, \diamond$ ). Overall, the performances are very similar to the ones obtained when the number of nodes is fixed for all subjects. 


\begin{tabular}{|c|c|c|c|}
\hline Interval $I_{q}$ & {$[5,13]$} & {$[14,22]$} & {$[5,21]$} \\
\hline \hline right HG & $0.43\left(18^{\star}, 18^{\diamond}\right)$ & $0.45\left(18^{\star}, 18^{\diamond}\right)$ & $0.45\left(18^{\star}, 18^{\diamond}\right)$ \\
\hline left HG & $0.35\left(18^{\star}, 12^{\diamond}\right)$ & $0.36\left(18^{\star}, 9^{\diamond}\right)$ & $0.36\left(18^{\star}, 11^{\diamond}\right)$ \\
\hline
\end{tabular}

Table 3. Mean G-SVC performances with variable $q$ over 18 simulations (number that resulted in accuracy above chance level $\star$, above the best benchmark method $\diamond$ )

Discussion. In this paper, we designed a graphical model and a graph-kernel that allows to compare and discriminate high dimensional patterns of fMRI activation across subjects. To our knowledge, this is the first time that a graph-based pattern recognition approach is used to study local fMRI activation patterns. Specifically, we have demonstrated the power of this approach in a classification task that aims at predicting experimental variables from fMRI patterns when the classifier is trained on data from a set of subjects and its generalization performance is tested on data from a different subject.

Our G-SVC framework performed above chance level in all cases, whereas none of the benchmark methods did. This validates that this framework is appropriate to deal with inter-subject variability that is not accounted for by a state of the art group alignment, such as the one offered in freesurfer. However, it is important to use the anatomical information at our disposal, as was attempted in this study by $i$ ) working on the cortical surface, and ii) defining a local coordinates system within our region of interest for each subject, thus using the most of the local individual anatomy. Indeed, we also tested G-SVC using the raw three-dimensional voxel coordinates for $x$, and the performances (not reported here) were systematically lower.

A major asset of using a graph kernel such as the one defined here is that the graphs can have different number of nodes, without having to strictly solve a graph matching problem, i.e to assign nodes to one another across instances. This is emphasized by the results of our simulations where the number of nodes was randomly chosen and forced to be different for each subject. In those, our framework still outperformed vector-based methods, demonstrating the ability to deal with graphs with different node numbers. We also noted a few simulations where the classification accuracy was higher than with a fixed $q$ for all subjects. This suggests that there might exist an "optimal" number of nodes for each subject and that performances could increase by adequately choosing $q$ for each subject. We will address this in future work, for instance by using a model selection criterion (BIC, AIC etc.) in the parcellation. Another source of potential improvement lies in selecting the hyper-parameter $\sigma_{f}$ and $\sigma_{g}$ of the kernel in a nested cross-validation, rather than using a heuristic estimator.

\section{Conclusion}

We have designed a graphical model carrying functional, geometric, and structural features to represent spatial patterns. With a custom-designed graph kernel providing a natural metric, this framework seems particularly attractive to perform classification tasks in multi-subject settings, as evidenced by the conclusive results obtained here when dealing with inter-subject functional variability in 
fMRI data. This modelling strategy therefore opens the possibility to characterize and discriminate populations based on their functional patterns of activation.

\section{Acknowledgment}

Thanks to the CNRS Neuro-IC program for funding, and to the Centre IRMf de Marseille and its staff for data acquisition.

\section{References}

1. G. Auzias, J. Lefèvre, A. Le Troter, C. Fischer, M. Perrot, J. Régis, and O. Coulon. Model-driven harmonic parameterization of the cortical surface. In Proc. 14th MICCAI, LNCS 6892 (Part II), pages 310-317, Toronto, Canada, Sep. 2011.

2. B. Caputo, K. Sim, F. Furesjo, and A. Smola. Appearance-based object recognition using svms: which kernel should i use? In Proc. of NIPS workshop on Stat. methods for computational experiments in visual processing and computer vision, 2002.

3. J. A. Clithero, D. V. Smith, R. M. Carter, and S. A. Huettel. Within- and crossparticipant classifiers reveal different neural coding of information. NeuroImage, 56(2):699 - 708, 2011. Multivariate Decoding and Brain Reading.

4. D. C. Van Essen and D. L. Dierker. Surface-Based and probabilistic atlases of primate cerebral cortex. Neuron, 56(2):209-225, October 2007.

5. G. Flandin, F. Kherif, X. Pennec, G. Malandain, N. Ayache, and J.-B. Poline. Improved detection sensitivity of functional MRI data using a brain parcellation technique. In Proc. 5th MICCAI, LNCS 2488 (Part I), pages 467-474, 2002.

6. T. Gärtner. Exponential and geometric kernels for graphs. In NIPS Workshop on Unreal Data: Principles of Modeling Nonvectorial Data, 2002.

7. D. Haussler. Convolution kernels on discrete structures. Technical Report UCSCCRL-99-10, UC Santa Cruz, 1999.

8. J. V. Haxby, M. I. Gobbini, M. L. Furey, A. Ishai, J. L. Schouten, and P. Pietrini. Distributed and overlapping representations of faces and objects in ventral temporal cortex. Science, 293(5539):2425-2430, 2001.

9. J. V. Haxby, J. S. Guntupalli, A. C. Connolly, Y. O. Halchenko, B. R. Conroy, M. I. Gobbini, M. Hanke, and P. J. Ramadge. A common, High-Dimensional model of the representational space in human ventral temporal cortex. Neuron, 72(2):404-416, October 2011.

10. C. Humphries, E. Liebenthal, and J. R. Binder. Tonotopic organization of human auditory cortex. NeuroImage, 50(3):1202 - 1211, 2010.

11. P. Mahé and J. P. Vert. Graph kernels based on tree patterns for molecules. Machine Learning, 75:3-35, 2009.

12. V. Michel, A. Gramfort, G. Varoquaux, E. Eger, C. Keribin, and B. Thirion. A supervised clustering approach for fMRI-based inference of brain states. Pattern Recognition, 45(6):2041 - 2049, 2012.

13. K. A. Norman, S. M. Polyn, G. J. Detre, and J. V. Haxby. Beyond mind-reading: multi-voxel pattern analysis of fMRI data. Trends in cognitive sciences, 10(9):424430, 2006.

14. T. Pavlidis. Structural pattern recognition. Springer-Verlag, 1977.

15. R. A. Poldrack, Y. O. Halchenko, and S. J. Hanson. Decoding the Large-Scale structure of brain function by classifying mental states across individuals. Psychological Science, 20(11):1364-1372, November 2009. 